\title{
XIV.
}

\section{Ueber die Aetiologie der experimentellen mycotischen Endocarditis.}

Nachschrift zu der vorstehenden Mittheilung des Dr. Wyssokowitsch.

Von Professor J. Orth in Göttingen.

Herr Dr. Wyssokowitsch hat sich in seiner Mittheilung über künstliche mycotische Endocarditis im Wesentlichen auf die Anführung der experimentell gewonnenen Thatsachen beschränkt, ohne auf eine Erörterung der allgemeinen Bedeutung dieser Thatsachen einzugehen. Bei Gelegenheit eines Vortrages in der pathologisch-anatomischen Section der Naturforscher-Versammlung in Strassburg ${ }^{1}$ ), wo ich über die Untersuchungen des Herrn Wyssokowitsch berichtete, habe ich bereits auf die Bedeutung dieser Experimente für die Lehre von den Krankheitsursachen, insbesondere für die Lehre von der Krankheitsdisposition aufmerksam gemacht, halte es aber bei der Wichtigkeit der Sache für angemessen, auch an diesem Orte und im unmittelbaren Anschlusse an die ausführliche Mittheilung des thatsächlichen Materiales eine kurze Besprechung der gewonnenen Resultate vorzunehmen.

Schon seit lange theilt man die Krankheitsursachen ein in äussere und innere, oder essentielle, eigentliche und prädisponirende Ursachen. Beide kamen bei unserer künstlichen Endocarditis zur Wirksamkeit, über beide haben die Untersuchungen werthvolle Aufschlüsse gewährt, welche freilich zunächst nur für diesen speciellen Fall Gültigkeit haben, welche aber doch auch über den engen Kreis der Experimente hinaus ihre grosse Bedeutung besitzen, indem sie zur Beleuchtung allgemeiner Gesetze dienen können.

Die eigentliche Krankheitsursache stellen in den Experimenten zweifellos die Mikroorganismen dar. Nur wenn sie in das

1) Tageblatt der 58. Versammlung deutscher Naturforscher und Aerzte. Strassburg 1885. S. 58. 
Blut eingeführt wurden, entstand die mycotische Klappen- und Gefässerkrankung und sowohl an den primär veränderten Theilen wie in den metastatischen Heerden fanden sich die Organismen in grosser Menge und zwar immer nur von solcher Art, wie sie in das Blut eingebracht worden waren. Freilich gelang es nicht, alle daraufhin geprüften Organismen zur Ansiedelung zu bringen, aber unter einer gewissen Anzahl verschiedener Formen hatte der Experimentator die Wahl; es lag ganz in seiner Willkür, welche von ihnen er zur Ansiedelung und zum Wachsthum bringen, durch welche er also die Krankheit erzeugt haben wollte. Ich sage allgemein die Krankheit, ich könnte auch specieller die Endocarditis und Endarteriitis sagen, denn darin stimmten alle überhaupt wirksamen Formen überein, dass sie Affectionen der Klappen und Arterienwand erzeugten, welche für die makroskopische und im Wesentlichen sogar für die mikroskopische Betrachtung keine Unterschiede darboten, als ïberzeugende Demonstration des Satzes, dass gleiche oder doch ganz ähnliche Erkrankungen durch ganz verschiedene Ursachen bedingt sein können. Geht man freilich in die Einzelheiten und zieht man insbesondere auch die Folgezustände der Herz- und Gefässerkrankungen in Betracht, so zeigen sich doch Unterschiede, welche wiederum zeigen, wie sehr doch der Charakter der Erkrankungen im Einzelnen von der besonderen Art, also von den besonderen Eigenschaften der Mikroorganismen abhängig ist, wobei allerdings nicht vergessen werden darf, dass auch dem Organismus, auf welchen die Mikroben einwirken, ein bestimmender Einfluss in dieser Beziehung zukommt. Endlich haben die Fxperimente ergeben, dass zur Entstehung der Klappenerkrankung eine gewisse Menge der Organismen nothwendig ist, dass also offenbar der Angriff derselben auf das Klappen- und Gefüssgewebe nur Erfolg hat, wenn derselbe gleichzeitig und an derselben Stelle von einer gewissen Anzahl der Organismen unternommen wird.

Viel wichtiger als diese Aufschlüsse über die directe Ursache der künstlichen Endocarditis sind meines Erachtens diejenigen, welche die Experimente über die disponirenden Ursachen derseiben ergeben haben. Je weiter wir forschen, desto mehr tritt uns, besonders bei den mycotischen Erkrankungen die Wichtig- 
keit der Disposition für die Entstehung der Krankheiten entgegen und die Erkenntniss von dieser grossen Bedeutung der Disposition erscheint mir sehr danach angethan, unsere an sich gewiss berechtigte Freude darüber, wie wir es doch in der Bakteriologie so herrlich weit gebracht, erheblich zu dämpfen. Mit der Auffindung pathogener Organismen bei einer Krankheit ist noch längst nicht genug geschehen, um ihre Aetiologie zu ergründen, denn dann bleibt immer noch die Frage nach den Dispositionen zu lösen. Erst wenu beide krankmachenden Factoren bekannt sind, erst dann and nur dans ist ein befriedigender Einblick in die Aetiologie und Genese der Erkrankung gewonnen. Wir sind durch diese Erkenntniss bei vielen und wichtigen Erkrankungen, ich erinnere an die Tuberculose und Phthise, auf dieselben Fragen zurückgekommen, welche schon vor Jahrzehnten erörtert wurden, freilich aber gehen wir von einem anderen und sicherlich günstigeren Standpunkte an ihre Bearbeitung heran, denn damals waren beide Krankheitsursachen unbekannt, die äusseren so gut wie die inneren, während jełzt doch wenigstens für eine Reihe wichtiger Erkrankungen die äusseren Ursachen in hinreichendem Maasse bekannt sind, um uns wichtige Anhaltspunkte für die Erforschung auch der disponirenden Ursachen zu gewähren. So ist für uns jetzt die Arbeit leichter und aussichtsvoller geworden, besonders auch dadurch, dass uns die Möglichkeit einer experimentellen Erforschung der Krankheitsdispositionen eröffnet ist, aber immerhin bleibt doch das Gebiet noch ein höchst schwieriges und verwickeltes und es wird noch einer langen und eingehenden Arbeit bedürfen, bis wir hier mit feststehenden, beweisbaren Thatsachen statt mit Wahrscheinlichkeiten und Hypothesen $z u$ rechnen haben.

Es ist nicht meine Absicht, mich hier in weitere allgemeine Erörterungen über die Art der Krankheitsdispositionen, über allgemeine und locale, angeborne und erworbene, dauernde und vorübergehende Dispositionen u. s. w. einzulassen oder eine allgemeine Besprechung der mechanischen, chemischen etc. Dispositionen vorzunehmen, sondern ich will nur in eine Besprechung unserer Experimente in dieser Richtung hin eintreten, da dieselben, wie ich meine, höchst wichtige Aufschlüsse ergeben haben, welche zunächst freilich nur für die experimentell erzengte 
Krankheit volle Gültigkeit haben, welche aber doch Ausblicke in andere Gebiete, auch der menschlichen Pathologie gestatten und deshalb auch als Bausteine für das künftig aufuführende Gebäude der Lehre von der Krankheitsdisposition Bedeutung haben.

Unsere Experimente zeigten nun also in erster Linie die grosse ätiologische Bedeutung der Disposition in schönster und kJarster Weise. Injection einer feinsten Aufschwemmnng geeigneter Organismen in's Blut von Kaninchen macht allein keine mycotische Endocarditis, die äussere Krankheitsursache ist also hier für sich allein, so lange sie auf ihre eigene Wirksamkeit angewiesen bleibt, nicht im Stande, die Krankheit zu erzeugen. Aber ebenso wenig ist auch die Disposition, die innere Krankheitsursache dazu im Stande, denn wir haben ebenso wie O. Rosenbach nach blosser Verletzung der Klappen gesunder Kaninchen niemals auch nur eine sichtbare Thrombose an den verletzten Stellen, geschweige denn eine Endocarditis entstehen sehen. Also beide Factoren sind jeder für sich allein ohnmächtig, dagegen entsteht sofort und wie es scheint mit vollster Regelmässigkeit (nur einmal liess die durchbohrte Klappe keine Veränderung erkennen, während gleichzeitig an der Aorta die gewöhnlichen mycotischen und thrombotischen Massen gefunden wurden) die Erkrankung, sobald beide in Gemeinschaft zur Einwirkung gelangen, und zwar entsteht nicht eine geringfügige Affection, sondern eine schwere Veränderung, welche zunächst zwar eine locale ist, aber bald weitere Organe in Mitleidenschaft zieht und den Tod des Thieres in kürzester Frist bedingt.

Noch wichtiger erscheint es mir, dass in unseren Experimenten "Disposition" nicht ein leerer Schall ist, ein Wort, dem die Begriffe fehlen, sondern dass wir mit einer greifbaren Thatsache rechnen können, denn wir haben es mit einer erworbenen localen Disposition zu thun, welche wir jederzeit und durchaus willkürlich herzustellen vermögen. Die Disposition ist keine dauernde, sondern eine relativ schnell vorübergehende, denn bereits 2 Tage nach geschehener Verletzung blieben einzelne vorgenommene Injectionen geeigneter Organismen erfolglos, die Disposition war bereits wieder verschwunden. Die Disposition wird erzeugt durch mechanische Einwirkungen, durch eine Verletzang. Es ist aber keineswegs nöthig, dass diese Verletzung eine schwere 
sei, denn die Organismen siedelten sich nicht nur an den durchstossenen Klappensegeln an, sondern auch an Stellen, wo die Sonde nur eben vorbeigeglitten war, also sicherlich nur minimale Verletzungen erzeugt haben konnte.

Nicht alle im Herzen gefundenen Veränderungen können als primäre angesehen werden, für deren Entstehung eine künstlich bewirkte Disposition angeschuldigt werden könnte, denn wir fanden - Herr Wyssokowitsch hat darauf auch bereits hingewiesen - ebenso wie das von der menschlichen mycotischen Endocarditis bekannt ist, Veränderungen, welche durch allmähliches Weitergreifen der Affection von einer zuerst ergriffenen Stelle aus per continuitatem oder per contactum entstanden sein mussten, bei welchen also ebenfalls locale Dispositionen vorhanden waren, aber solche, welche durch die schon bestehende Affection selbst erzeugt waren. Möglicherweise könnten auf diese Weise auch die so regelmässigen Affectionen der Bicuspidalis erklärt werden, obgleich andererseits nicht ausser Acht zu lassen ist, dass auch diese Klappe besonders nach der Durchstossung der Aortensegel von dem Sondenknopf getroffen werden musste. Es kann eine solche Erklärung aber nicht mehr Platz greifen für die mycotischen Veränderungen in der Carotis und Aorta, denn abgesehen davon, dass dieselben oft von den Klappenveränderungen räumlich ganz getrennt waren, haben wir solche auch vorgefunden, wo eine gröbere Verletzung, eine Klappenzerstörung gar nicht zu Stande gekommen war ${ }^{1}$ ). Es muss hier also in der That die leichte oberflächliche Reibung der Intima mittelst der Sonde genïgt haben um jene localen Veränderungen am Gewebe zu erzeugen, welche nothwendig sind, damit die Organismen da sich festsetzen und wachsen können.

Es fragt sich nun, welcher Art sind diese durch das leichte Trauma bewirkten Gewebsstörungen und wie können sie die Ansiedelung der Organismen ermöglichen? Es ist nicht wohl anzunehmen, dass etwa erzeugte Unebenheiten oder Thrombenbildungen an der Oberfläche diese Wirkung besässen, denn in den Controlexperimenten wurden überhaupt keine Thrombenbildungen an den verletzten Stellen gesehen und die durch das

1) Solcher Experimente habe ich später noch mehrere mit bestem Erfolge angestellt. 
Trauma direct erzeugten unregelmässigen Rissflächen bestehen an den Klappen wenigstens nach 2 und mehr Tagen noch ebenso wie am ersten Tage und doch vermögen die Organismen dann nicht mehr zu haften. Diese Thatsache wird nicht geändert durch die Annahme, dass die Organismen Blutveränderungen erzeugten, kleinste Gerinnungen, Haufenbildungen farbloser Blatkörperchen, welche als Vehikel der Mikroben dienen und ihre Anlagerung an den Klappen begünstigen könnten '), denn diese müssten doch bei der Injection der Organismen einige Tage nach der Operation in ganz gleicher Weise zur Wirksamkeit kommen wie bei früherer Injection und doch bleibt dann die Wirkung aus. Also um eine rein mechanische Wirkung, um eine mechanische Begünstigung des Haftens der im Blute kreisenden Organismen kann es sich nicht wohl handeln, sondern es wird kaum etwas anderes übrig bleiben, als anzunehmen, dass das Trauma eine Ernährungsstörung an dem Gewebe, in erster Linie wohl an den Endothelzellen, vielleicht auch einen kleinen Endotheldefect bewirkt und dass dadurch in dem Kampf der Pilzzellen mit dem Gewebe resp. den Gewebszellen an dem verletzten Orte die Widerstandsfähigkeit der Gewebe herabgesetzt wird, so dass nun die Pilze den Sieg erringen, festen Fuss fassen, wachsen und unaufhaltsam immer weiter in das Gewebe vordringen können. Es mag dabei begünstigend für die erste Anlagerung der Organismen wirken, dass auch Veränderungen im Blute durch sie entstanden sind, dass vielleicht kleine Leucocytenklümpchen gleich eine grössere Menge von Organismen auf einmal an eine bestimmte Stelle bringen, aber das kann das Wesen der Vorgänge nicht ausmachen, denn diese Leucocytenklümpchen müssten doch überall hin im gesammten Gefässsystem gelangen können, während doch die Ansiedelung der Organismen nur an ganz wenigen und an ganz bestimmten Stellen stattfindet, nehmlich nur da, wo die traumatische Einwirkung eine Gewebsstörung bewirkt hat. Hier liegt die locale Disposition; die ich möchte sagen functionelle Gewebsstörung ist unerlässlich dazu, dass die Ansiedelung der Organismen vor sich gehen könne.

Durch die Annahme einer solchen Gewebsstörung wird es auch erklärlich, warum die locale Disposition eine so schnell

1) v. Recklinghausen, l. e. S. 60 (Discussionsbemerkung). 
vurübergehende ist. Es könnten sich bis zum zweiten Tage die in ibrer Ernährung gestörten Endothelien resp. Gewebszellen wieder erholen, es könnte ein kleiner Defect derselben durch Regeneration wieder ausgeglichen, das zerrissene Klappengewebe mit Endothel überzogen werden, so dass die frühere Widerstandsfähigkeit des Gewebes etwa durch die Wiederherstellung einer schützenden Endotheldecke in kürzester Zeit wiedergewonnen wäre.

Das Alles ist vorläufig - vielleicht gelingt es mir bei den fortgesetzten Untersuchungen auch dafür eine thatsächliche Grundlage zu finden - nur Hypothese, ein Erklärungsversuch und für nichts Weiteres gebe ich es aus, aber es wird, mag die Erklärung richtig oder falsch sein, damit an den thatsächlichen Resultaten der Experimente nichts geändert, welche ich noch einmal dahin kurz zusammenfasse, dass bei unserer Versuchsanordnung zur Entstehung der mycotischen Klappen- und Gefässerkrankung eine locale Disposition nöthig war, welche durch ein relativ geringfügiges Trauma willkürlich und leicht, aber nur vorübergehend für eine kurze Zeit hergestellt werden konnte.

Es stehen anscheinend mit den gewonnenen Resultaten in Widerspruch die Ergebnisse der Untersuchungen, welche Ribbert mit einer der auch von Wyssokowitsch benutzten Mikrobenarten (Staphylococcus) an der gleichen Thierart (Kaninchen) angestellt und über welche derselbe gleichfalls auf der Naturforscherversammlung in Strassburg ${ }^{1}$ ) berichtet hat. Ribbert iujicirte in die Ohrvene von Kaninchen Kartoffelculturen von Staphylokokken in einer mit kleinen Kartoffelpartikelchen hergestellten Emulsion und erzielte damit regelmässig multiple myocarditische Heerde and in einem kleineren Theile der Fälle auch Endocarditis, obwohl doch im Gegensatze zu unseren Experimenten keinerlei traumatische Einwirkung auf das Herz stattgefunden hatte. Wenn auch bei Ribbert die Endocarditis nur eine untergeordnete Rolle gegenüber der Myocarditis spielte, während bei uns umgekehrt die Endocarditis (resp. Arteriitis) die regelmässige Erscheinung, die Myocarditis nur Nebenbefund war, so würde immerhin die Möglichkeit, ohne sonstige Eingriffe 
durch Injection von Staphylokokken in's Blut bei Kaninchen eine Endocarditis za erzeugen, den Werth unserer Versuchsergebnisse beeinträchtigen, wenn nicht eine Erklärung des Widerspruchs gefunden werden könnte. Eine solche ergiebt sich aber leicht aus der ganz verschiedenen Versuchsanordnung in beiden Experimentalreihen. Während wir die Organismen in feinstvertheilter Form in das Blut einbrachten, hat Ribbert sich einer Emulsion bedient, d. h. er hat von vornherein Embolien gemacht. Damit aber hat er sofort eine locale Disposition für das Wachsthum der Organismen erzeugt, gleichgültig, ob man diese darin sucht, dass den Organismen durch die Kartoffelpartikelchen das Haftenbleiben an gewissen Stellen erleichtert wurde oder darin, dass durch die Embolien locale Circulations- und Ernährungsstörungen bedingt wurden, welche die Widerstandsfähigkeit des Gewebes herabsetzten. Es mag beides gelegentlich seine Rolle spielen, jedenfalls glaube ich aus unseren Experimenten die Bedeutung embolischer Störungen in letzterem Sinne erschliessen zu können.

Nachdem wir die Endocarditis erzeugt hatten, bestand ja bei uns insofern eine ähnliche Versuchsanordnung wie bei Ribbert, als die beobachteten metastatischen Heerde in Nieren, Milz, Darm, Leber, Gehirn nicht mehr in Folge einer von uns willkürlich erzengten localen Disposition entstanden, sondern auf embolischem Wege von der Primäraffection aus erzeugt wurden, wobei die Embolie selbst erst die locale Disposition bewirkte. Am besten ist das bei den Streptococcusexperimenten zu sehen, wo grosse anämisch-nekrotische Infarcte in den Nieren entstanden. Hier, insbesondere in den Randpartien der Heerde, also jedenfalls da, wo durch die Ernährungsstörung die Widerstandsfähigkeit des Gewebes erheblich herabgesetzt war, war es zu so mächtigen Wucherungen der Mikroben gekommen, dass nicht nur ganze Glomeruli, sondern noch weit ausgedehntere intertubuläre Capillargebiete nach der Färbung mit Methylenblau wie mit Berlinerblau injicirt aussahen. Und das waren Organismen, welche, wie Dr. Wyssokowitsch gelegentlich anderer Untersuchungen sich zu wiederholten Malen überzeugte, selbst wenn sie in grossen Massen in das Blut gespritzt wurden, nicht im Stande waren, auch nur einen einzigen sichtbaren localen Krank- 
heitsheerd zu erzeugen, subald sie in feiner Vertheilung eingeführt wurden.

Bei den Staphylokokken mag in den Nieren ein wesentlich mechanisches Moment, das leichtere Haften, bei der Entstehung der metastatischen Heerde eine Rolle spielen, da sie auch schon bei ihrer Anwesenheit in fein vertheilter Form im Blute im Stande sind, in den Nieren Krankheitsheerde zu erzeugen. Aber in den übrigen Organen können sie das nicht, da muss erst eine locale Störung vorhanden sein, damit sie überhaupt sich festzusetzen und zu wachsen vermögen. Eine solche hat aber Ribbert erzengt, indem er durch seine Emulsion multiple kleine Embolien bewirkte und so erklärt es sich unschwer, dass er so regelmässig myocarditische embolische Heerde beobachtete. Die zuweilen entstandene Endocarditis kann aber sowohl secundär durch Fortleitung aus einem myocarditischen Heerd hervorgegangen als auch primär aus einer Embolie etwa vorhandener Klappengefässe entstanden sein. Jedenfalls wird man aus dem Umstande, dass nur in einem kleineren Theil der Fälle die Endocarditis gefunden wurde, schliessen dürfen, dass in diesen besondere begünstigende, also disponirende Ursachen vorlagen, welche nur relativ selten zur Wirkung gelangten. In unseren Experimenten war die Endocarditis durchaus unabhängig van Myocarditis und nichts deutete darauf hin, dass die Endocarditis oder Arteriitis etwa aus Embolien in Vasa vasorum hervorgegangen seien. Schon die Localisation der Primärerkrankung im Allgemeinen, nur an den Klappen und an den Aorten- resp. Carotiswandungen, ferner die Localisation im Besonderen, nur an den verletzten Theilen, dann aber auch der histologische Befund sprechen gegen eine solche Annahme. Selbst wenn wir von der Frage, ob denn überhaupt an all den erkrankten Stellen Gefässe, in welchen eine Embolie stattgefunden haben könnte, vorhanden waren, absahen, so sprach das Fehlen embolisirter Gefässe, das Wachsthum der Organismen zunächst an der Oberfläche der Häute, das deutliche Vordringen der Organismenmassen von innen nach aussen in den Gefässwandungen, das von innen nach aussen fortschreitende Auf- und Abblättern der elastischen Membranen der Media zu deutlich dafür, dass die Organismen aus dem kreisenden Blute an der Oberfläche sich angelagert hatten, als dass man darau 
hätte zweifeln können. Damit aber eine solche oberflächliche Ansiedelung stattfinden kounte, dazu war es nothwendig, zuvor eine locale Disposition zu erzeugen, wie wir es durch die angebrachte Verletzung gethan haben.

Wenn all das, was ich über die Disposition gesagt habe, einzig und allein für unsere experimentell erzeugte Kaninchenkrankheit Gültigkeit hätte, so würde dadurch immerhin ein interessanter und wichtiger Aufschluss über die Genese der Krankheiten wenigstens für einen bestimmten Fall gewonnen sein. Ich glaube aber, dass man es wohl unternchmen darf, die so gewonnene Erkenntniss auch für den entsprechenden Fall aus der menschlichen Pathologie zu verwerthen.

Bei einem gar nicht kleinen Procentsatz der Fälle von mycotischer ulceröser Endocarditis handelt es sich um eine sog. recurrirende Endocarditis, d. h. die Mycose hat ein schon von früher her verändertes, also doch wohl grade dadurch disponirtes Gewebe ergriffen. In anderen Fällen gesellt sich die Endocarditis secundär zu mycotischen, häufig puerperalen Erkrankungen hinzu, ohne dass die Klappen von fruher her verändert waren. Da geschieht es in der Regel, dass die mycotischen Prozesse an denjenigen Stellen beginnen, wo die Klappensegel bei ihrem Schluss an einander schlagen und ich sehe in unseren Experimenten eine thatsächliche Stütze für die von Virchow schon seit langer Zeit immer wieder gegebene Erklärung, dass es dieses mechanische Moment des Aneinanderprallens der Segel sei, welches eine Prädisposition für die Entstehung der Affection grade an dieser Stelle bewirkte. Ist gar, wie es Virchow für die chlorotischen Veränderungen im Circulationsapparat ausgeführt hat, in Folge angeborner Verhältnisse eine abnorme Spannung im arteriellen Gefässsystem vorhanden, so wird erst recht diese mechanische Wirkung zur Geltung kommen können: Häufigkeit der puerperalen Endocarditis bei Chlorotischen. Es mag dabei hinzukommen, und ich möchte darauf entschieden Werth legen, dass durch die der Endocarditis vorausgehende Blutveränderung die Endothelien bereits in ihrer Ernährung gestört worden sind - Anhaltspunkte für diese Annahme haben wir genügend - und dass sie dadurch gegenüber der erwähnten mechanischen Einwirkung noch empfindlicher geworden sind. Wir haben aber in 
unseren Experimenten gesehen, wie geringfügig die Störung zu sein brauchte, um den im Blate vorhandenen Organismen die Ansiedelung zu ermöglichen. An den Klappenschlusslinien könnte die Ansiedelung allerdings auch noch dadurch befördert werden, dass die isolirten oder in kleinsten Fibrin- oder Leucocytenklümpchen enthaltenen Organismen mechanisch an und in das Klappengewebe gepresst werden.

Bleiben also auch der Möglichkeiten und der Zweifel noch genug übrig, so ist immerhin, wie ich meine, durch den thatsächlichen Nachweis der grossen Bedeutung der localen und zwar hier traumatischen Disposition für eine bestimmte Form mycotischer Endocarditis bei 'Thieren auch für das Verständniss der menschlichen mycotischen Endocarditis eine etwas sicherere Grundlage gewonnen.

\section{Nachtrag.}

Die fortgesetzten Untersuchungen haben bis jetzt wenigstens das eine Resultat schon ergeben, dass in Folge der einfachen Verletzung an der Kaninchenaorta proliferative Veränderungen entstehen, da in einem Falle 41 Stunden, in einem zweiten 48 Stunden nach der Operation zahlreiche Mitosen an Endothelzellen, eine geringere Zahl auch an anderen Zellen der Gefässwand gefunden wurden. Damit hat die Annahme, es möchte die Abnahme der localen Disposition mit reparatorischen Veränderungen der Gefässwand, besonders des Endothels zusammenhängen, sowie die andere, es möchte die Disposition überhaupt in Störungen der Gefässwandelemente, in erster Linie wieder des Endothels, beruhen, eine neue Stütze erhalten. 